\title{
Redshift-distance relations from type la supernova observations
}

\section{New constraints on grey dust models}

\author{
A. R. Robaina ${ }^{1,2, \star}$ and J. Cepa ${ }^{2,3}$ \\ 1 Facultad de Física, Universidad de La Laguna, 38071 La Laguna, Tenerife, Spain \\ e-mail: arobaina@iac.es \\ 2 Instituto de Astrofísica de Canarias, 38200 La Laguna, Tenerife, Spain \\ 3 Departamento de Astrofísica, Facultad de Física, Universidad de La Laguna, 38071 La Laguna, Tenerife, Spain \\ e-mail: jcn@iac.es
}

Received 27 March 2006 / Accepted 3 August 2006

ABSTRACT

\begin{abstract}
Extinction due to intergalactic grey dust has been proposed as an alternative to accelerated expansion to account for the dimming of SnIa fluxes beyond $z \simeq 0.5$. The "replenishing" grey dust model, although fitting the observational data, does not seem to be based on physical assumptions. For this reason, in this paper we propose a new grey dust model whose dust distribution follows the comoving SFR density evolution, a reasonably established phenomenon. This new model is compared with the updated photometric data sample from the High Z Supernova Search Team (HZT). Also, using pairs of supernovae at different redshifts, the possibility of any "patchy" distribution proposed to explain the discrepancies between the "high $z$ " dust model and the SnIa observations at very high redshift $(z \geq 0.9)$ is ruled out. Finally, the data are compared with different models with and without dark energy and a best fit to a universe with adimensional parameters $\Omega_{\mathrm{m} 0}=0.31$ and $\Omega_{\Lambda 0}=0.69$ is obtained, from which an age of the universe of $t_{0} \simeq 14.6 \times 10^{9}$ years is derived. This age is compatible with the age of the globular clusters using an equation of state $\omega=-1$ and it obviates the need to resort to any kind of phantom energy.
\end{abstract}

Key words. cosmological parameters - cosmology: theory - distance scale - galaxy: abundances

\section{Introduction}

After the discovery by Riess et al. (1998) and Perlmutter et al. (1999) of the excessive weakness of type Ia supernovae (SnIa) at high redshift, cosmology has undergone a revolution similar to the cosmic microwave background (CMB) discovery. The cosmological interpretation of these observations led to the postulation of the accelerated expansion of the universe as a consequence of a "dark energy" of negative pressure and unknown origin. Given the difficult physical interpretation of this dark energy, either in terms of a cosmological constant, quintessence, or even some form of phantom energy, several mechanisms have been proposed to explain SnIa observations without resorting to an accelerated expansion. Among them are models of intergalactic grey dust, which try to explain the dimming of the observed brightness at $z \simeq 0.5$ by assuming that an absorbent medium exists in intergalactic space whose extinction does not depend on wavelength. These dust models have been driven to evolve at the same rate as the data supplied by SnIa teams.

The first models, proposed by Aguirre (1999a,b), were based on both a model of dust formed by carbon needles and a Draine \& Lee dust model (Draine \& Lee 1984), obtaining a density parameter of $\Omega_{\text {Dust }} \sim 10^{-5}$. Also, Goobar et al. (2002) have developed a model of intergalactic dust whose effects on the distance modulus vs. $z$ are indistinguishable from those caused by a $\Lambda C D M$ universe with $\Omega_{\mathrm{m} 0} \simeq 0.3, \Omega_{\Lambda 0} \simeq 0.7$ (more information on the intergalactic medium amount and its effects can be found in, for example, Bianchi \& Ferrara 2005; Inoue \& Kamaya 2003; Nath et al. 1999).

* Currently at the Max-Planck-Institut für Astronomie, Heidelberg, Germany; e-mail: arobaina@mpia-hd.mpg.de
In any case, grey dust models imply universes with $\Omega_{\mathrm{m} 0}=1$ or non-Euclidean geometries. The first possibility can be discarded by the $2 \mathrm{dF}$ experiment (Peacock et al. 2001), while the second can be rejected by analysis of the anisotropies observed in the CMB (Spergel et al. 2003), from which a total parameter $\Omega=1$ is derived.

Apart from grey dust models, another effect that could hamper the need for an accelerated expansion universe to explain SnIa data is their luminosity evolution. Observational evidence shows SnIa luminosity variations with galactic morphology (Hamuy et al. 1996, 2000; Branch et al. 1996; Reindl et al. 2005), while some theoretical studies (Hoflich et al. 1998, 2000) suggest that SnIa luminosity could have some dependence on redshift. Were this true, this would question the status of standard candle for this kind of objects. In any case, these studies are beyond the scope of this article and are merely mentioned as further possibilities in addition to grey dust models in order to avoid accelerated expansion.

In this paper we present observational evidence to refute the high- $z$ grey dust model even in the case of patchy distribution. We also propose a new grey dust model with a density distribution following that of the star formation rate (SFR) density. This SFR-based distribution is based on observational data, thus making the grey dust model based on physical assumptions that best fits the supernova data. In Sect. 2 we give an overview of existing theoretical grey dust models, including high- $z$ and the replenishing grey dust. In this section the new SFR-based distribution model is also developed. In Sect. 3 we derive the dust-free model by best-fitting the SnIa data, obtaining its density parameters and the age of the universe, and compare the data with dust models, including the one developed here. We also explore the 
possibility of reconciling the observational data with the high- $z$ dust model by means of a patchy dust distribution. Finally, we draw our conclusions in Sect. 4.

\section{Theoretical models}

The existence of grey dust would have an enormous impact on the analysis of the High Z Supernova Search Team (HZT) and the Supernova Cosmology Project (SCP) observations, returning the standard model of the universe to the point where it was before 1998 with their contradictions of the CMB anisotropy experiments, which point to a flat universe $(\Omega=1)$. This is the reason a detailed study of the different possibilities is required.

Any intergalactic dust model producing grey extinction relevant to the study of SnIa observations must have the following characteristics:

1. large grain size, to account for the achromaticy of the absortion and to comply with the mechanism of preferential destruction of smaller size grains (Aguirre 1999b);

2. an efficient expulsion mechanism in the galaxy where the grains are formed. It must explain why only this type of dust is found in the IGM;

3. coherence with the expected metallicity of the intergalactic medium;

4. the dust distribution must be based on a hypothesis in turn based on reasonably established physical observables.

\subsection{High-z grey dust}

Even though Aguirre's (1999a,b) high- $z$ dust model implies a uniform dust distribution similar to that of non-relativistic matter,

$\rho_{\text {dust }}=\rho_{\text {dust }}^{0}(1+z)^{3}$,

which fulfils the first three of the four requirements mentioned above, the latest results published by the HZT (Riess et al. 2004), with SnIa data beyond $z=1.5$, almost certainly (at the $4 \sigma$ level) reject the possibility of this high- $z$ dust model. Figure 1 shows the large deviation of the Aguirre (1999a,b) model in the high- $z$ region compared to the experimental data and the best fit.

However a patchy dust distribution could render this conclusion unsound. If the intergalactic dust distribution were not homogeneous, it might be possible that the light coming from an SnIa at high redshift $(z \geq 0.9)$ propagates through more or less absorbent medium, depending on the source position in the sky. The deviation of the observed data from the high- $z$ dust model would then be due to a bias produced by the grey dust, leaving the magnitude of the absorbed supernovae below the detection limit of current telescopes. In this case, the sample observed at very high redshift would belong to the group of SnIa whose optical path has not been very affected by the aforementioned absortion, and for this reason its apparent brightness would be higher than that inferred by supposing a completely homogeneous dust distribution.

The inhomogeneity in the grey dust distribution is a solid enough possibility to take it into account in spite of Aguirre's (1999a,b) arguments. In his theoretical study, possible mechanisms for preferential ejection of grey dust from the galaxies and the temporal scale on which it could have been homogeneously distributed, are explained. They are partly corroborated by the work of Renzini (1997) on the metallicity of the intracumular

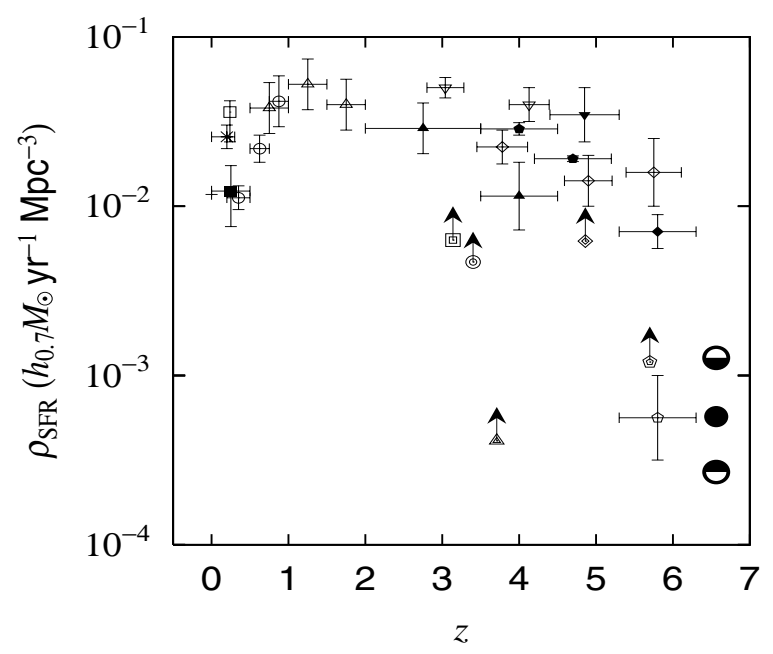

Fig. 1. Comoving $\rho_{\mathrm{SFR}}$ as function of redshift. Figure taken from Taniguchi et al. (2005).

gas. Nevertheless, it is difficult to explain how a dust distribution formed by baryonic matter can escape from the baryonic large-scale structures observed in the universe. Aguirre himself (1999a) suggests that the study of systematic differences between the flux received from SnIa hosted by field and cluster galaxies could distinguish between a universe in accelerated expansion and an effect due to grey dust, respectively, by restricting implicitly the homogenity to the intracumular space.

\subsection{Replenishing grey dust}

As different surveys reached more and more distant redshifts, it became evident that the high- $z$ dust model was unable to explain the data. However, the reasonable values obtained by Aguirre (1999a,b) for the time scale of the expulsion mechanisms of his grain models have promoted the birth of new models that fit the data much better. A good example is the replenishing grey dust model, proposed by Goobar et al. (2002), whose match to the data is at least as good as the $\Omega_{\Lambda} \neq 0$ models.

This replenishing grey dust model is based on a grey dust extinction that follows a density distribution of the form:

$\rho_{\text {dust }}=\rho_{\text {dust }}^{o}(1+z)^{a}$,

where

$a=\left\{\begin{array}{l}3 \text { if } z<0.5 \\ 0 \text { if } z>0.5\end{array}\right.$

This model requires extremely efficient formation and expulsion of the dust in the range $z>0.5$ so that the dilution due to the expansion of the universe is exactly compensated by the metal formation in galaxies and its further removal. However, this behaviour is not consistent with the current knowledge of the overall star formation episodes that took place in the universe. As a consequence, a physical explanation of this replenishing mechanism is still lacking. Moreover, the hitherto unjustified sharp transition from $a=0$ to $a=3$ represents a dramatic end of metal formation and expulsion from the galaxy without a transition era.

\subsection{SFR-based model}

To avoid these features of the replenishing dust model, a grey dust model based on the star formation rate (SFR) density evolution is proposed here. It will be shown that the proposed model 


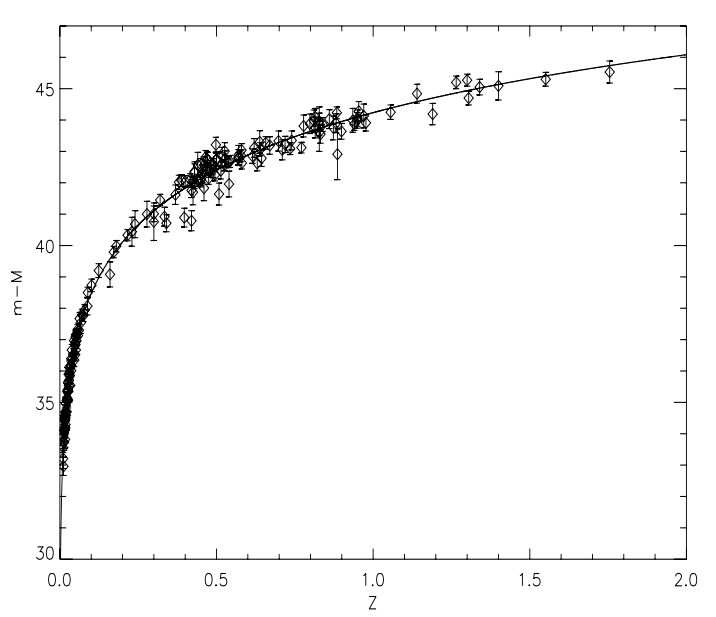

Fig. 2. Hubble diagram. Distance moduli of observational SnIa data vs. $z$. Overplotted are the $\Omega_{\mathrm{m} 0}=0.31, \Omega_{\Lambda 0}=0.69$ universe, which is the best fit for a flat cosmology obtained from $\chi^{2}$ statistics.

fits the existing SnIa data equally well, while at the same time providing a dust production based on the current knowledge of star formation evolution in the universe.

Following the results compiled by Taniguchi et al. (2005) on the comoving SFR density evolution (Fig. 2), it can be observed that the comoving SFR density $\left(\rho_{\mathrm{SFR}}^{\mathrm{c}}\right)$ is approximately constant in the range $1 \leq z \leq 2$. In the region $0<z<1$, the SFR density may be reasonably represented by a simple relation with redshift, with lower SFR at recent times. For simpler description, the SFR density curve is then represented by these two regimes. For our purposes this simple parameterization is adequate, but see Oda $\&$ Totani (2005) for more complex parameterization.

In this context, the comoving SFR density is represented by

$\rho_{\mathrm{SFR}}^{\mathrm{c}} \propto\left\{\begin{array}{lll}10^{1+z} & \text { if } & 0<z<1 \\ \text { constant if } & 1<z<2 .\end{array}\right.$

The evolution of the SFR proper density $\left(\rho_{\mathrm{SFR}}^{\mathrm{p}}\right)$ is

$\rho_{\mathrm{SFR}}^{\mathrm{p}}(z) \propto \int_{z_{i}}^{z} \frac{\rho_{\mathrm{SFR}}^{\mathrm{c}} \cdot(1+z)^{1 / 2}}{a_{0}^{3} \cdot H_{0}} \mathrm{~d} z$,

where $a_{0}$ is the scale factor at present and the value taken for $H_{0}$ is $65 \mathrm{~km} \mathrm{~s}^{-1} \mathrm{Mpc}^{-1}$.

Nevertheless, although the dust density derives from the SFR evolution, not all the dust escapes from the galaxy to the IGM. At least a fraction of the metals produced will be retained in low mass stars that never reach the core collapse supernova phase and do not present strong stellar winds. An estimate of the retained metal fraction can be obtained from the Salpeter initial mass function (Salpeter 1955) from $0.1 M_{\odot}$ to $8 M_{\odot}$. This SFR-based distribution will then include, as explained above, a transition at $z \simeq 1$ between the two regimes of maximum and decreasing SFR:

$$
\begin{aligned}
& \rho_{\text {dust }} \propto \\
& \begin{cases}\rho_{\text {dust } 1}^{0}\left(-\frac{\sqrt{\pi}}{2 \ln 10^{3 / 2}} \operatorname{Erfi}(\sqrt{1+z} \sqrt{\ln 10})+\frac{10^{1+z} \sqrt{1+z}}{\ln 10}\right) & \text { if } z<1 \\
\rho_{\text {dust2 }}^{0}\left(\frac{2}{3}(1+z)^{3 / 2}\right) & \text { if } z>1 .\end{cases}
\end{aligned}
$$

The constants $\rho_{\text {dust } 1}^{0}$, and $\rho_{\text {dust } 2}^{0}$, which include the estimation of metals retained in low mass stars, must be fitted in the region $(0<z<1)$ to match the expected deviation at $z=0.5$, while they are chosen to guarantee the continuity of the curve in the region $z>1$.

Although the dust is produced by the star formation, the relevant physical magnitude to evaluate is the opacity, not the SFR. The resulting extinction is then delayed with respect to dust production owing to the time interval elapsed from production to insertion into the IGM. This delay can be estimated from assumptions for the velocity of the ejecta.

In order to fulfil the above-mentioned requirements, this grey dust model must present grain characteristics similar to those proposed in previous models, reproducing the expected values for $R_{v}$ and absorptions (Goobar et al. 2002). Finally, the injection mechanism and the preferential destruction of small size grains will be assumed to be those discussed by Aguirre (1999b, 2001).

\section{Results}

\subsection{Cosmological constraints}

In this work we have used the latest published HZT data (Riess et al. 2004; Strolger et al. 2004), since it represents the most significant sample with photometric values to date. This sample has greatly benefited from the results of the Great Observatories Origins Deep Survey (GOODS) project, carried out using the Advanced Camera for Surveys (ACS) on board the Hubble Space Telescope (HST). The SnIa classification in the groups Gold, Silver, and Bronze used is explained in full detail by Strolger et al. (2004). The most certain classification is the Gold class, characterised by a clear spectroscopic confirmation of the supernova type.

In this section, the observational data are fitted by a model composed of non-relativistic matter and dark energy, neglecting the contributions of radiation and relativistic matter, which do not play a significant role in the universe in the redshift range considered. The magnitude used for the fit is based on the distance modulus,

$m-M=5 \log D_{\mathrm{L}}+25$,

where $m$ and $M$ are the apparent and absolute magnitudes, respectively, and $D_{\mathrm{L}}$ is the luminosity distance in Mpc. Assuming a flat geometry, as derived from the spectra of the CMB anisotropies, we have obtained, via an $\chi^{2}$ criterion, the best fit with the parameters $\Omega_{\mathrm{m} 0}=0.31$ and $\Omega_{\Lambda 0}=0.69$, values similar to those found in previous studies (Riess et al. 1998, 2004; Perlmutter et al. 1999), and consistent with those derived from $2 \mathrm{dF}$ results (Peacock et al. 2001).

These values are only indicative, given the fit dependence with the rejecting criterion. In Fig. 2 we show the Hubble diagram of the entire sample, together with the best fit found.

It is worth noting that these values of mass and darkenergy density parameters, assuming a Hubble constant of $65 \mathrm{~km} \mathrm{~s}^{-1} \mathrm{Mpc}^{-1}$, yield an age of the universe around $t_{0} \simeq$ $14.6 \times 10^{9}$ years as derived using Cepa (2004), with the constraint $\omega=-1$ for the equation of state of dark energy (i.e. a cosmological constant). This result is very nearly compatible with the globular cluster age of $12.9 \pm 2.9$ Gy found by Carretta et al. (2000) and its time of formation, without resorting to a phantom energy (Cepa 2004), although the error margin is still too large to determine the equation of state of the dark energy. 


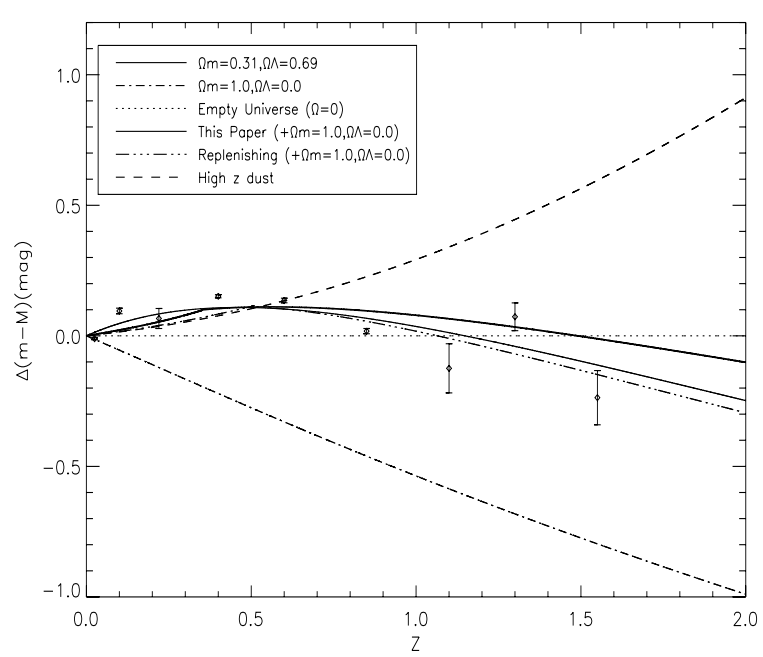

Fig. 3. Averaged SnIa data and theoretical models showing the modulus difference of distances with respect to a universe in constant expansion $(\Omega=0)$. The high- $z$ dust model is rejected at $4 \sigma$ (dashed line). The best fit is given by an $\Omega_{\mathrm{m} 0}=0.31, \Omega_{\Lambda 0}=0.69$ universe (solid thin line) with no grey dust contribution. The SFR-based dust model represented takes into account a delay of $2.7 \mathrm{~Gy}$ between the dust formation in the disk and its homogeneous distribution in the IGM.

\subsection{The new dust model}

The SnIa weakness at $z \simeq 0.5$ and their excess brightness at $z>0.5$, representing the transition from an accelerated to a decelerated universe, are best appreciated by representing not the distance moduli but the distance moduli differences with respect to the empty Milne's universe in constant expansion.

The grey dust models are represented as the distance moduli of a flat universe with $\Omega_{\mathrm{m} 0}=1, \Omega_{\Lambda 0}=0$, with an atenuation obtained by

$\Delta m_{\text {dust }}=-2.5 \log \left\{\exp \left(C \int_{0}^{z} \rho_{\text {dust }}(z) r(z) \mathrm{d} z\right)\right\}$

where $r(z)$ is the comoving distance travelled by the SnIa light, $\rho_{\text {dust }}(z)$ is given by Eqs. (1), (2) or (3), and $C$ is a constant used for dimensional purposes.

In the case of the SFR-based dust model proposed in the present work (3), it will be necessary to include a delay between dust formation and its incorporation into the IGM to contribute to the observed extinction. To estimate the time interval in which the dust ejected could reach a homogeneous distribution in the intracluster space, we assume an expulsion velocity for the dust grains of around $1000 \mathrm{~km} \mathrm{~s}^{-1}$, following Aguirre et al. (2001) and Shustov \& Vibe (1995). A delay of $\Delta t \approx 3 \times 10^{9}$ years is obtained, implying that the homogeneity of the dust produced at $z \simeq 1$ is reached around a redshift of $0.3<z<0.4$.

Figure 3 shows the different universe models, together with the weighted average data. From this figure it can be concluded that the high- $z$ dust model can be discarded to a confidence level of $4 \sigma$ for a homogeneous dust distribution. Also, it can be observed that the differences between the $\Omega_{\mathrm{m} 0}=0.31, \Omega_{\Lambda 0}=0.69$ universe and the replenishing dust model are almost negligible. Finally, the differences between our model with respect to the SnIa data are smaller than $1 \sigma$. As discussed in Sect. 2, the advantage of the new model is that the dust distribution derives from current observational evidence.
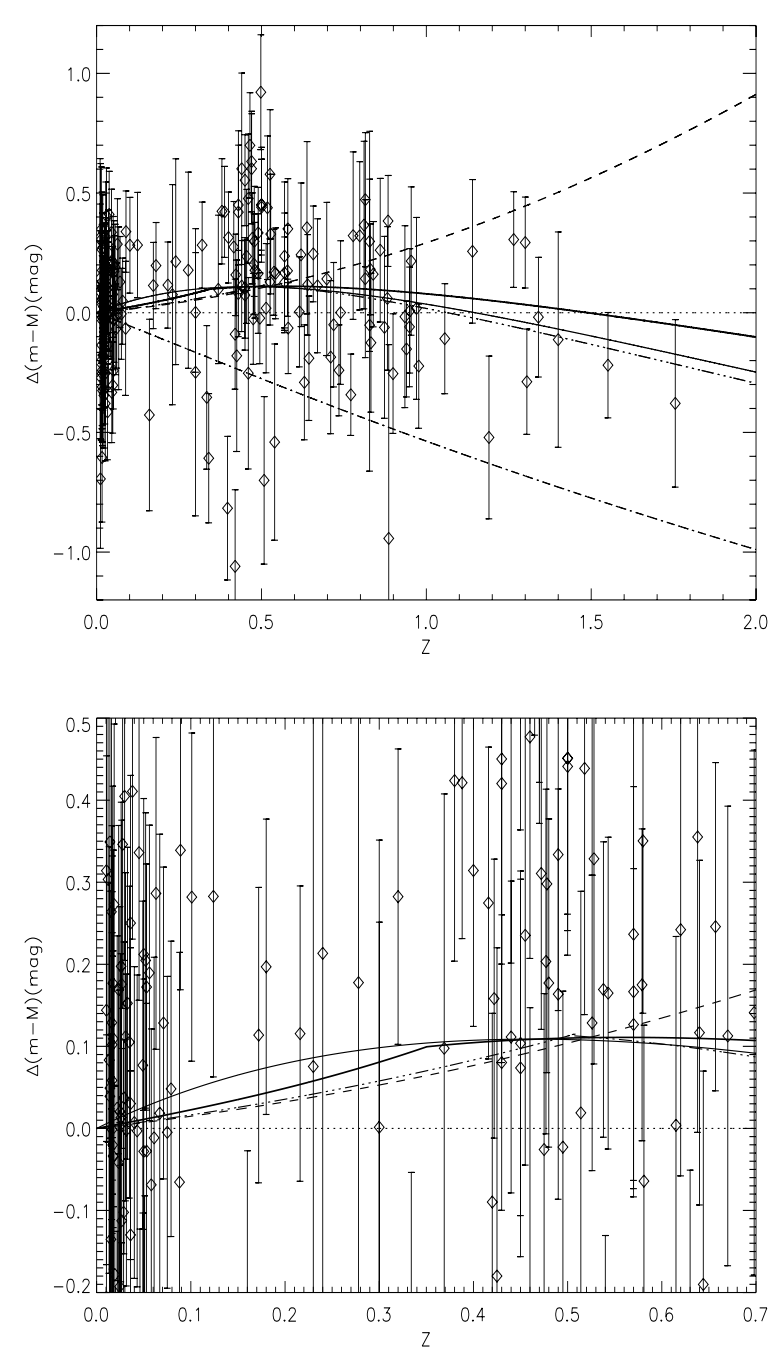

Fig. 4. Top: as in Fig. 3, but representing the whole SnIa sample. The SFR-based distribution fits the data within the error bars of individual data. Bottom: detail of the $0<z<0.7$ region.

In Fig. 4 the entire sample of photometric data from SnIa is shown superposed on the theoretical models considered so far. It can be seen that the error bars of individual photometric data include both the SFR-based dust and the "replenishing" dust models. This model is quite flexible: the ambiguity in current cosmic star formation history studies (expulsion velocities, delay times and SFR curve parameterization) does not have a big impact on our results, especially if the constants are empirically fitted as explained in Sect. 2.3.

In all these universe models (the grey dust ones), we have assumed a flat geometry with $\Omega_{\mathrm{m} 0}=1.0$, but this assumption contradicts the results of $2 \mathrm{dF}$ (Peacock et al. 2001). For this reason we have also developed an open universe $\left(\Omega_{\mathrm{m} 0}=0.3\right.$, $\left.\Omega_{\Lambda 0}=0.0\right)$ with SFR-based dust whose differences are less than $1 \sigma$ compared to the SnIa data (Fig. 5). The implications of this open universe will be discussed in Sect. 4.

\subsection{Further evidence against high-z grey dust}

It might be argued that a non-homogeneous grey dust distribution could produce the deviation from the experimental data compared to Aguirre's model. According to this hypothesis, at 
high redshifts only the SnIa whose line of sight to the observer passes through more voids (in other words: whose "beam" is emptier) can be observed, since the others are too absorbed by the dust. In this way, distant SnIa are brighter than expected according to the high- $z$ dust model, lying somewhere between the high- $z$ grey dust and the $\Omega_{\mathrm{m} 0}=1$ model, where the data points are located (Fig. 4).

To evaluate the feasibility of this hypothesis, the SnIa sample is searched for close pairs of SnIa with very different redshifts, in which the low $z(z \simeq 0.5)$ member of the pair is fainter than expected from the Milne model, while the high- $z$ one $(z \geq 0.9)$ is not. Were such a pair to be found, it should be concluded that inhomogeneities in the high- $z$ grey dust model cannot be deemed responsible for the observed SnIa distance modulus distribution.

Assuming a Robertson-Walker metric, the angle subtended by an object in a flat universe is

$\theta=\frac{D_{\mathrm{T}}(z)}{D_{\mathrm{P}}(z)}$,

where $D_{\mathrm{T}}$ is the transversal distance and $D_{\mathrm{P}}$ the proper distance. From this equation, and assuming a cosmological model, the angle corresponding to a transversal distance can be evaluated.

To minimize the influence of photometric and model errors when applying this test, it is convenient to look for apparent SnIa pairs of very different redshifts, of which the SnIa at low $z$ show the highest brightness dimming. This maximum SnIa dimming is observed around $z=0.5$ (Riess et al. 1998, 2004; Perlmutter et al. 1999). Assuming a characteristic size of $30 \mathrm{Mpc}$ for a void, the angle corresponding to this distance at $z=0.5$ will represent the highest projected angular distance to search for a suitable pair to apply this test. This is a rather stringent requirement since a void size of $30 \mathrm{Mpc}$ is smaller than those found by Einasto et al. (1994). From their data, an average diameter of around $\overline{\Phi_{\text {void }}} \simeq 91 \mathrm{Mpc}$ is deduced. In Table 1 , the angles corresponding to this distance at $z=0.5$ for different models of universe are shown.

With these hypotheses, the requirements to apply this test to supernovae pairs are the following:

1. the two members of the candidate pair have to be in the ranges $0.35<z<0.65$ for the low $z$ and $z \geq 0.9$ for the high $z$, respectively. This requirement maximizes the difference in brightness of the pair members to reduce the contribution from photometric errors as much as possible;

2. for the same reason, the difference in redshift between the members of the pair must be $\Delta z \geq 0.4$;

3. the angular distance on the sky between the two members of the pair must be lower than the value of $\simeq 1$ degree assumed in Table 1;

4. both supernovae must be classified with the maximum confidence as type Ia. This requirement restricts the search to the Gold sample.

For such a small SnIa sample (157 SnIa ) it might seem difficult to fulfil these requirements. Luckily, the systematic search for high- $z$ supernovae has centred on specific regions of the sky, mainly on the GOODS project HDFN and CDFS fields. The sky distribution of the SnIa sample is represented in Fig. 6. The whole sample is shown in the left panel, while on the right the SnIa found in the HDFN are shown, demonstrating the small distances that separate the SnIa in these deeply studied regions.

Table 2 summarises the results of our study for different cosmologies. Here, all the SnIa at $0.35 \leq z \leq 0.65$ that are members

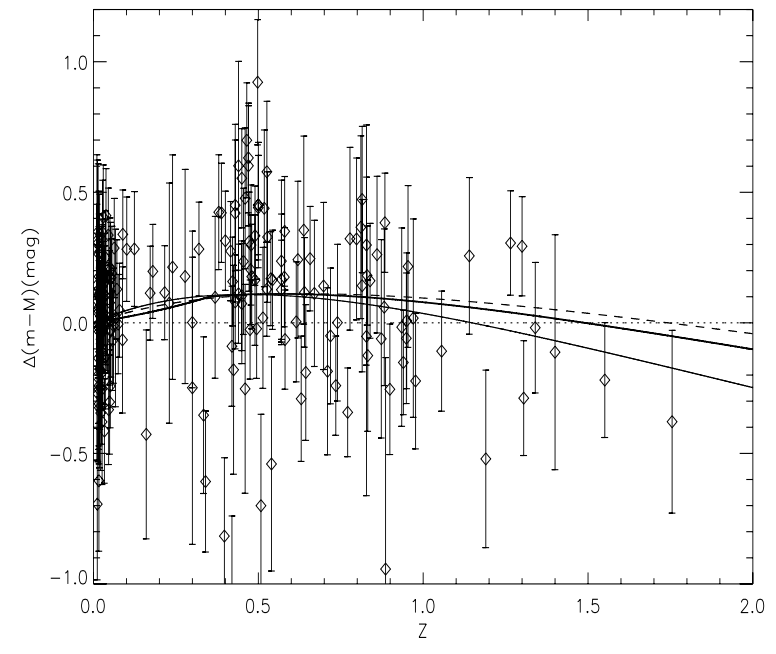

Fig. 5. Differences with respect to an empty universe. The flat (thick line) and open (dashed line) SFR-based dust universe models are within the $1 \sigma$ confidence level.

Table 1. Values for the angle (in degrees) subtended by a $D_{\mathrm{T}}$ of $30 \mathrm{Mpc}$ at $z=0.5$ in different universes, with the assumed Hubble constant of $65 \mathrm{~km} \mathrm{~s}^{-1} \mathrm{Mpc}^{-1}$.

\begin{tabular}{ccc}
\hline \hline$\Omega_{\mathrm{m} 0}$ & $\Omega_{\Lambda 0}$ & $\theta$ \\
\hline 0.3 & 0.0 & 0.9502 \\
0.3 & 0.7 & 0.8451 \\
1.0 & 0.0 & 1.0155 \\
\hline
\end{tabular}

Table 2. Number of SnIa pairs found with an angular distance between members lower than the angle $\theta$ given by Table 1 for the corresponding cosmology. A: pairs in which both members belong to the Gold sample. B: at least one of the members belong to the Silver sample.

\begin{tabular}{ccccccc}
\hline \hline$\Omega_{\mathrm{m} 0}$ & $\Omega_{\Lambda 0}$ & $z_{1}$ & $z_{2}$ & Angle & Gold (A) & Silver (B) \\
\hline 0.3 & 0.0 & $0.35-0.65$ & $\geq 0.9$ & $\theta$ & 15 & 5 \\
0.3 & 0.0 & $0.35-0.65$ & $\geq 0.9$ & $\theta / 2$ & 15 & 3 \\
0.3 & 0.7 & $0.35-0.65$ & $\geq 0.9$ & $\theta$ & 15 & 5 \\
0.3 & 0.7 & $0.35-0.65$ & $\geq 0.9$ & $\theta / 2$ & 15 & 2 \\
1.0 & 0.0 & $0.35-0.65$ & $\geq 0.9$ & $\theta$ & 15 & 5 \\
1.0 & 0.0 & $0.35-0.65$ & $\geq 0.9$ & $\theta / 2$ & 15 & 3 \\
\hline
\end{tabular}

of a pair are weaker than expected in an empty universe. A relatively large number of pairs is found, considering that the whole sample is composed by only 186 SnIa.

\section{Conclusions}

With the available SnIa data we have calculated the best-fit dustfree universe model, obtaining $\Omega_{\mathrm{m} 0}=0.31, \Omega_{\Lambda 0}=0.69$. These results are very similar to those found in previous studies of luminosity distance to type Ia supernovae, leading to a reconciliation of the age of globular clusters with the age of the universe $\left(t_{0} \simeq 14.6 \times 10^{9}\right.$ years, as derived from the model, when assuming a cosmological constant-like dark energy, $\omega=-1$ ). Phantom energies can thus be avoided, as long as the mean values do not increase. However, the uncertainties in the age of globular clusters are still too great to constrain the equation of state of dark energy. 

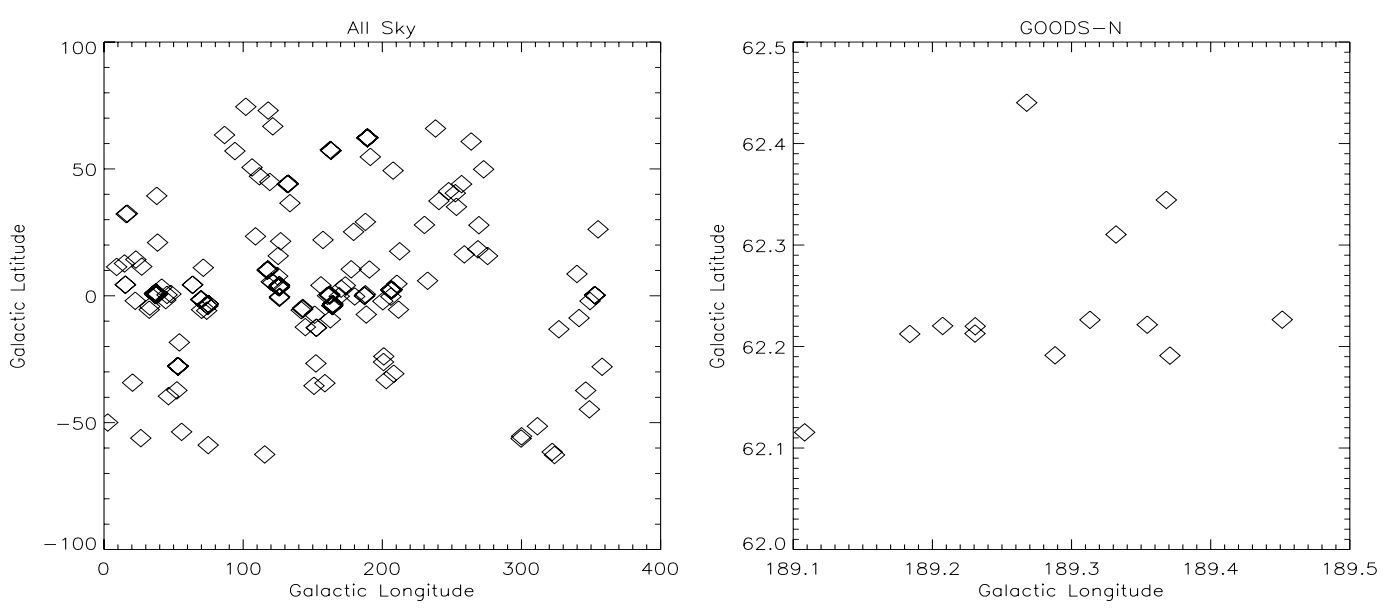

Fig. 6. Location on the sky of the SnIa sample. Left: position of all the supernovae in the sample. Right: detail of the Hubble Deep Field North region. The units on the axis are degrees.

A new grey dust model based on replenishing dust but including the observed comoving SFR density distribution has been derived, thereby providing an alternative model based on observational evidence. The extinction produced by this new SFR-based grey dust with $\Omega_{\mathrm{m} 0}=1.0$ is very close to the $\Omega_{\mathrm{m} 0}=0.31, \Omega_{\Lambda 0}=0.69$ model with no extinction. Apart from the difficulties in providing the mass required for a flat universe and the age conflict arising therefrom, the models would be distinguished only by future supernovae surveys at high $z$ with higher photometric accuracy, such as those to be carried up with SNAP (Supernova Acceleration Probe) (Aldering et al. 2002) and with the JWST (James Webb Space Telescope, Stockman et al. 1998), which could provide the evidence that will help to decide between a currently dark energy-dominated universe and a matter-dominated one. Moreover, the next generation SnIa surveys, with more accurate data, will be essential because the uncertainties in the current SnIa data, due to the contributions of approximations, photometric errors, luminosity maximum estimations at high $z$, etc., impose several restrictions on the validity of the cosmological results. Only when combined with other experiments such as CMB anisotropies or gravitational lenses is it possible to derive more stringent conclusions.

All the flat grey dust models presented here consider Euclidean geometry with an adimensional mass parameter, $\Omega_{\mathrm{m} 0}=1$, a hypothesis against the data obtained so far (Peacock et al. 2001). Even with the results of del Popolo (2003), who has suggested that current $\Omega_{\mathrm{m} 0}$ values obtained from clusters of galaxies are underestimated by $20 \%$, a parameter $\Omega_{\mathrm{m} 0}=1$ is excluded from the acceptable margins. For this reason we have included an open model $\Omega_{\mathrm{m} 0}=0.3, \Omega_{\Lambda 0}=0.0$ with our SFR-based grey dust attenuation. Although the confidence level of this model with respect to the SnIa data is below $1 \sigma$, it introduces a serious conflict with the interpretation of the WMAP results (Spergel et al. 2003). To reconcile this model with the CMB anisotropies experiment it is necessary to resort to exotic mechanisms, such as thermalization of the radiation due to the intracumular dust at high $z$.

The high- $z$ dust model is rejected out to $4 \sigma$ even if the grey dust presents a patchy distribution, as shown by the pair test devised here. However, most of the data at very high redshift corresponds to GOODS fields, which cover a small area on the sky (Fig. 5). Pairs at other positions on the sky combined with high-accuracy photometry would allow us to ascertain whether there is any significant amount of grey dust in the universe. Nevertheless, the consistency of the standard model and the weak basis for the alternative explanations makes the possibility of having an open model describing our universe difficult.

Acknowledgements. We wish to thank G. A. Tammann, A. Tortosa Andreu, and A. M. SÃąnchez Quintana for helpful comments. This work was supported by the Spanish Plan Nacional de Astronomía y Astrofísica under grant AYA2005-04149. This research made use of HZT SnIa photometric data, NASA's Astrophysics Data System (ADS), and the Nasa/IPAC Extragalactic Database (NED).

\section{References}

Aguirre, A. N. 1999a, ApJ, 512, L19

Aguirre, A. N. 1999b, ApJ, 525, 583

Aguirre, A. N., Hernquist, L. S. Joop, et al. 2001, ApJ, 561, 521

Bianchi, S., \& Ferrara, A. 2005, MNRAS, 358, 379

Aldering, G., et al. 2002 [arXiv: astro-ph/0209550]

Branch, D., Romanishin, W., \& Baron, E. 1996, ApJ, 465, 73 (Erratum, 467)

Cepa, J. 2004, A\&A, 422, 831

del Popolo, A. 2003, A\&A, 408, 43

Draine, B., \& Lee, H. 1984, ApJ, 285, 89

Einasto, M., Einasto, J., Tago, E., Dalton, G. B., Andernach, H., et al. 1994, MNRAS, 269, 301

Fujita, S., Ajiki, M., Shioya, Y., et al. 2003, ApJ, 586, L115

Goobar, A., Bergstrom, L., \& Mortsell, E. 2002, A\&A, 384, 1

Hamuy, M., Phillips, M. M., Suntzeff, N. B., et al. 1996a, AJ, 112, 2391

Hamuy, M., Trager, S. C., Pinto, P. A., et al. 2000, AJ, 120, 1479

Hoflich, P., Dominguez, I., Thielemann, F. K., Wheeler, J. C., et al. 1998, LIACo, 34,243

Hoflich, P., Nomoto, K., Umeda, H., \& Wheeler, J. C. 2000, ApJ, 528, 590

Inoue, A. K., \& Kamaya, H. 2003, MNRAS, 341, L7

Nath, B. B., Sethi, S. K., \& Shchekinov, Y. 1999, MNRAS, 303, 1

Oda, T., \& Totani, T. 2005, ApJ, 630, 59

Peacock, J. A., Cole, S., Norberg, P., et al. 2001, Nature, 410, 169

Perlmutter, S., Aldering, G., Goldhaber, G., et al. 1999, ApJ, 517, 565

Reindl, B., Tammann, G. A., Sandage, A., \& Saha, A. 2005 [arXiv: astro-ph/0501664]

Renzini, A. 1997, ApJ, 488, 35

Riess, A. G., Filippenko, A. V., Challis, P., et al. 1998, AJ, 116, 1009

Riess, A. G., Strolger, L.-G., Tonry, J., et al. 2004, ApJ, 607, 665

Salpeter, E. E. 1955, ApJ, 121, 161

Shustov, B., \& Vibe, D. 1995, Astron. Rep., 39, 578

Spergel, D. N., Verde, L., Peiris, H. V., et al. 2003, ApJS, 148, 175

Stockman, H. S., Stiavelli, M., Im, M., Mather, J. C., et al. 1998, ASPC, 133, $24 \mathrm{~S}$

Strolger, L. G., et al. 2004, ApJ, 613, 200

Taniguchi, Y., Ajiki, M., Nagao, T., et al. 2004 [arXiv:astro-ph/0407542] 